\title{
VALORES Y ACTITUDES DE LOS JOVENES ANTE EL TRABAJO *
}

\author{
Enric Sanchis
}

Universidad de Valencia

RESUMEN. Se ofrece un estado de la cuestión razonado de la literatura española, italiana y francesa, analizando las actitudes y valores de los jóvenes que han traspasado el umbral de la enseñanza obligatoria y buscan su primer empleo. El trabajo como valor va cediendo terreno al trabajo como instrumento, pero las actitudes de rechazo son minoritarias.

El paro juvenil es una de las consecuencias más graves de la crisis económica actual, así como uno de los elementos que la diferencian de la de 1929 , cuando la edad no era una variable tan discriminante al respecto. Varias disciplinas han intentado analizarlo desde diferentes perspectivas. Por el lado de la demanda de trabajo, los enfoques económicos tratan sobre todo de dar una explicación a la contracción del nivel de empleo. Por el lado de la oferta, demografía, sociología y psicología social estudian la evolución de la fuerza de trabajo, sus actitudes ante la actividad económica y las repercusiones que tiene sobre el individuo el ocupar una u otra posición en el mercado de trabajo.

Los valores y actitudes de los jóvenes ante el trabajo se han convertido

* Este artículo forma parte de una investigación sobre paro juvenil, financiada por la Institució Valenciana d'Estudis i Investigació, que se encuentra ya en fase avanzada de redacción. 
en la coletilla de las interpretaciones específicas del paro juvenil. Aparte de la incidencia de la demografía, la evolución de las tasas de actividad o la inadecuada formación, serían los propios jóvenes quienes con sus elevadas exigencias, el rechazo de la disciplina laboral y su escasa motivación hacia el trabajo acabarían autoexcluyéndose del empleo.

Esta manera de enfocar la cuestión es muy popular entre nosotros y no sólo en medios empresariales, y tampoco sólo entre nosotros. Durante cierto tiempo, en Italia tuvo mucho éxito la frase de Amendola «los jóvenes que no tienen ganas de trabajar»; y en Francia, hace ya más de diez años, un libro de Rousselet ${ }^{1}$, que casi podría calificarse de clásico en la materia, explicaba con detalle los procesos que habían conducido a esta situación y se convertía en referencia obligatoria de muchas investigaciones posteriores, aunque sólo fuera - como de hecho ha ocurrido en no pocas ocasiones- para refutarlo.

En las páginas que siguen se pretende ofrecer un estado de la cuestión razonado, aunque seguramente no exhaustivo, de la literatura española, italiana y francesa sobre el tema, si bien a veces se hacen incursiones fuera de este ámbito. Se ha procedido de esta manera para evitar establecer comparaciones entre situaciones que sólo cobran pleno significado en relación con el sistema cultural en que se insertan. Instituciones como el salario mínimo garantizado para los schools leavers en busca de primer empleo o el mismo modelo familiar dominante hacen arriesgado confrontar sin matizaciones datos sobre jóvenes españoles y británicos o daneses, por ejemplo, mientras que la comparación parece menos aventurada cuando existe cierta continuidad cultural, como suponemos que ocurre entre los países que constituyen el ámbito aludido.

Al hablar de jóvenes nos estamos refiriendo no tanto a unas determinadas cohortes de edad como a ese amplio colectivo que ha traspasado más o menos satisfactoriamente el umbral de la enseñanza obligatoria y que, provisto de unos estudios secundarios completos o no, se supone que debe intentar buscarse la vida en esa gruesa franja del mercado de trabajo donde no están casi ninguna de las ocupaciones nobles y muy pocas de las más degradadas: la de una gran parte de los empleos industriales y terciarios, de cuello azul y de cuello blanco ${ }^{2}$. Desde luego, así el campo no queda delimitado con demasiada precisión, pero tampoco quienes tildan a los jóvenes de haraganes suelen afinar los conceptos. En los límites —aunque cada vez resulta más difícil trazarlosquedarían, por arriba, los titulados universitarios, a quienes se supone más legitimados para rechazar durante más tiempo los empleos mediocres e intentar la caza de una ocupación noble; por abajo, los fracasados escolares, con menos capacidad de elección y sólo legitimados para aceptar lo que se les

\section{' J. Rousselet, L'allergie au travail, Seuil, París, 1974.}

2 La situación de los jóvenes cercanos al medio rural merece consideración aparte. Cfr., al respecto, VV. AA., Sociedad rural y juventud campesina, Ministerio de Agricultura, 1985. 
ofrezca. Así pues, en realidad vamos a ocuparnos sobre todo de las actitudes y valores, por una parte, de los jóvenes originarios de clase media y con estudios secundarios cuyo tradicional mercado de trabajo (burocracias industriales, de servicios y públicas), además de estrecharse, por sí mismo y por la competencia de los titulados superiores, se ha deteriorado como consecuencia de los procesos de descualificación provocados por el cambio tecnológico; y, por otra, de los jóvenes originarios de clase obrera que ya no comienzan a ir al trabajo de la mano de su padre y saliendo de hogares miserables, sino de la escuela obligatoria, y para los cuales el ganarse un jornal ya no es cuestión de supervivencia.

Puestos a generalizar, puede defenderse sin temor a equivocarse que la afirmación de que este tipo de jóvenes actuales rechazan el trabajo $-y$, por tanto, que el mecanismo de socialización a través del trabajo se ha roto- es esencialmente falsa. A lo sumo es un fenómeno de minorías. Si lo que se afirma, más matizadamente, es que el trabajo ha dejado de ser un valor central y cada vez más es contemplado, sobre todo, en sus aspectos instrumentales, es decir, como medio para conseguir otras cosas (lo que acabaría incidiendo sobre la productividad y la calidad de la prestación laboral), entonces la cuestión se complica bastante, pues datos empíricos y diversas líneas interpretativas llevan a conclusiones no convergentes.

Dejando aparte los discursos moralistas y los ideológicamente interesados, los análisis de las actitudes y valores de los jóvenes — sobre todo cuando parten de la hipótesis de que son nuevos o diferentes- se enfrentan a una dificultad metodológica inicial no siempre bien resuelta: con qué o quién compararlos. ¿Con los jóvenes de la generación anterior?, ¿con los coetáneos adultos?, ¿con un «antes» idílico en el que había conciencia profesional, esperanza en la promoción, amor al trabajo y satisfacción en la obra bien hecha?

Parece muy dudoso, por otra parte, que pueda hablarse de una homogeneidad, de una ideología juvenil al respecto. La fuerte influencia del medio social de pertenencia sobre las mentalidades de los jóvenes, por ejemplo, pone en serios aprietos la tesis de la existencia de una subcultura juvenil. Una investigación francesa sobre jóvenes todavía poco o nada tocados por la crisis del empleo llegaba a la siguiente conclusión: «En el análisis que hemos realizado de las diversas opiniones relativas al trabajo, el éxito o el fracaso, nada permite detectar el reflejo de una ideología juvenil homogénea que se opondría total o parcialmente a la de los adultos» ${ }^{3}$. Es más - continúan estos autores-, pueden darse diferencias más profundas en el seno del grupo juvenil que las que puedan existir entre éste y el grupo adulto. $\mathrm{Y}$ el pretendido rechazo ge-

${ }^{3}$ VV. AA., «L'idée de travail, de réussite et d'echec chez les jeunes de milieux sociaux et scolaires différents», en Cabiers du Centre d'Etudes de l'Emploi, núm. 7, 1975 , p. 95. 
nérico del trabajo no sólo es muy discutible, sino que, aun cuando exista a nivel de aspiraciones, en la práctica no se manifiesta. Los jóvenes estudiados daban la impresión de estar más cerca de los puntos de vista propios de una madurez desencantada que de los de una juventud apasionada. Manifestaban, eso sí, una visión instrumental del trabajo, que era considerado como una actividad más entre otras muchas; o sea, como los adultos, se concluye. Las apreciaciones de Yankelovich sobre la juventud obrera estadounidense de la misma época van paralelas. Para estos jóvenes, en 1973, un empleo debería reunir los requisitos principales siguientes: que el trabajo sea interesante, que esté bien pagado y que permita desarrollar las capacidades personales ${ }^{4}$. Nada del otro mundo, como se ve. Para el autor, las exigencias y opiniones de los jóvenes en relación con el trabajo no parecen ni demasiado nuevas ni descabelladas; quizás se diferenciarían de los adultos en el grado de optimismo respecto a la posibilidad de conseguir lo deseado.

\section{Casi nadie trabaja por gusto}

No estaría de más recordar aquí que trabajo procede etimológicamente de tripalium, palabra que designaba un artilugio con tres pies al que se sujetaba al condenado para someterlo a tormento, y que en toda la tradición cultural judeo-cristiana la idea de trabajo aparece las más de las veces relacionada con la de padecimientos. Como se sabe por el Génesis, el trabajo irrumpe por vez primera en nuestro imaginario colectivo cultural en tanto que castigo, vinculado al pecado original y a la expulsión del Edén, donde no había que trabajar para sobrevivir. Y si bien es cierto que ya San Pablo lo elevó a la categoría de obligación moral (quien no trabaje que no coma), aunque esto debe tener también algún tipo de relación con la escasa eficacia laboral del modo de producción esclavista, tiempo después Santo Tomás matizaría hábilmente que la máxima paulina se dirigía a la comunidad en su conjunto, no al individuo, no particularmente a quien pueda vivir con lo que tiene sin trabajar; y el trabajo vuelve a ser considerado en esencia como una actividad que degrada.

Gracias a Max Weber conocemos muy bien el proceso a través del cual, impulsado por las consecuencias prácticas de la ética calvinista, el trabajo deviene el valor central en la vida del individuo; sólo que esta novedad no es más que una excepción en la historia de la humanidad que difícilmente ha tomado cuerpo en los países de tradición dominante católica, sobre todo en el nuestro, donde ésta se ha combinado con una industrialización tardía y un desarrollismo intenso que ha premiado más la sagacidad especulativa que el

'D. Yankelovich, The New Morality. A Profile of American Youtb in the 70's, McGraw Hill, Nueva York, 1974, pp. 30-31. 
esfuerzo por la obra bien hecha y la capacidad profesional, donde la cultura tradicional conserva una concepción señorial del trabajo que desprecia el esfuerzo físico e incluso el intelectual cuando es por cuenta ajena ${ }^{5}$. Y cuando del plano ideológico descendemos al más pedestre de la vida laboral de cada día, las construcciones idílicas alrededor de la idea de trabajo salen todavía peor libradas. El encargado de relaciones humanas de cualquier gran empresa sabe cuán cierto es que no menos del 90 por 100 de sus empleados se aburren soberanamente y que esto crea problemas, si no graves, permanentes a la organización. Como ha señalado Cuvillier, por lo que se ve, parece que el trabajo propiamente dicho sólo puede ser una obligación desagradable, fuente de tedio y de empobrecimiento del ser, un mal necesario para ganarse la vida; el mundo del trabajo es un mundo en el que el trabajo no es de la incumbencia del trabajador y del que está excluido el placer producido por la ocupacion, como experiencia profesional vivida y como objetivo válido en sí mismo ${ }^{6}$.

Los jóvenes se socializan no sólo a partir de los mensajes edificantes que interesadamente se les dirige, sino también de aquéllos menos explícitos que contiene el sistema cultural que los envuelve $\mathrm{y}$, sobre todo, de las prácticas que observan en sus coetáneos. $\mathrm{Y}$ el hecho es que los valores que se supone deberían gobernar sus actitudes frente al trabajo nunca fueron demasiado centrales. No sería de extrañar, pues, que no mostraran excesivo entusiasmo a la hora de incorporarse a la vida activa. Pero tanto la falta de ahínco laboral de los jóvenes como las quejas patronales sobre los perjuicios correspondientes no constituyen fenómenos nuevos. Cottereau, que ha tenido la feliz ocurrencia de estudiar la literatura patronal francesa del siglo XIX, ha detectado que ya entonces existían situaciones de «rabiosa actualidad»: «Ya en 1848, la Cámara de Comercio de París explicaba que el aprendizaje artesanal estaba acabado. La juventud de París había perdido el gusto por el trabajo. Prefería ganar el dinero aprisas ${ }^{7}$. Y de algunas narraciones obreras de la época se deduce la misma actitud instrumental frente al trabajo, una falta de ambiciones profesionales que trasluce en realidad una falta de ilusión sobre lo que les espera; fenómeno que «constituye precisamente uno de los rasgos más característicos y más constantes de los hijos de los obreros, por muy lejos que se llegue en la historia» ${ }^{8}$. Así, pues, «a lo largo del capitalismo industrial, tanto en el caso de los muchachos como en el de las muchachas, parece que el trabajo obrero no ha sido contemplado jamás como un fin en sí mismo, suscep-

5 Véase, en este sentido, M. BELTRÁN, «La subcultura juvenil», en VV. AA., Informe sociológico sobre la juventud española 1960/1982, SM, Madrid, 1984; particularmente, p. 184 .

- R. Cuvillier, «El trabajo y los trabajadores intelectuales ante las ideas y la práctica sociales», en Revista Internacional del Trabajo, vol. 89, núm. 4, 1974.

7 A. Cottereau, «Les jeunes contre le boulot: une histoire vieille comme le capitalisme industriel», en Autrement, núm. 21, 1979, p. 197.

s Ibid., p. 198. 
tible de polarizar las esperanzas de la mayoría. Una cosa es estar obligado al trabajo asalariado y otra adherirse ideológicamente al mismo" ${ }^{9}$. Si ha habido una evolución en el campo de los valores y actitudes - concluye Cottereau-, el tema a analizar sería más bien cómo a medida que la descualificación del trabajo se extiende y afecta a nuevas actividades en el sector terciario, se va colocando en el centro de la gravedad, gana terreno y hace escuela la vieja filosofía de los jóvenes obreros frente al trabajo. Filosofía que, hay que insistir, no denota tanto rechazo-boicot como una concepción instrumental del trabajo.

Romagnoli, que ha estudiado las relaciones de los jóvenes con el trabajo en el marco de una investigación IARD sobre la condición juvenil en Italia, se muestra muy reticente respecto a los «nuevos» valores y actitudes con que se supone que éstos se incorporan a la vida activa. La desconfianza no deriva sólo de que investigaciones ya antiguas sobre los jóvenes del 68 se encontraron con que las actitudes y las imágenes tradicionales seguían sustancialmente en vigencia, sino sobre todo «del hecho de no haber encontrado nunca una demostración convincente sobre la "centralidad" del trabajo para quien no es joven», $y$, por lo tanto, de la sospecha de que mucho de lo que se ha dicho sobre la crisis del trabajo tiene una gran carga ideológica. A quien afirma que los jóvenes comienzan a manifestar una relación «diferente» con el trabajo habría que preguntarle: ¿diferente respecto a qué otra posible relación?, ¿respecto al orgullo de un oficio que ya no existe?, ¿respecto al «instrumentalismo" del obrero de las sociedades «opulentas» de que ya hablaban las investigaciones de los años sesenta? La única posible relación «diferente» con el trabajo debería ser su rechazo masivo. Pero contra el trabajo no se ha producido nada de este tipo, si acaso contra este trabajo, lo cual no es precisamente una novedad ${ }^{10}$.

Cuando se dice que el trabajo es un «instrumento» — continúa Romagnoli- se está haciendo una afirmación muy ambigua, porque en el fondo nadie o casi nadie ha sostenido nunca que el trabajo sea un fin en sí mismo. «En definitiva, cuando el trabajo deja de ser una "profesión" para convertirse en un "puesto", y deja de ser un "deber" para convertirse en un "derecho", ya no se puede seguir ocultando su naturaleza intrínseca de necesidad social.» Y si el trabajo es una necesidad, la denuncia de la «desafección» (que ha existido siempre y se ha expresado siempre como «alergia» a la explotación) se torna claramente moralizante. La novedad, que no afecta sólo a los jóvenes, sino a todo aquel que deba trabajar para vivir, reside probablemente en el desarrollo de una actitud «racional» frente al trabajo similar a la que se ha

Ibid., p. 205.

${ }^{10} \mathrm{G}$. Romagnoli, «Il lavoro e i suoi significati», en VV.AA., Giovani oggi, Il Mulino, Bolonia, 1984, p. 53. 
producido respecto al ejercicio del derecho de voto. «La extensión de los derechos de ciudadanía introduce en el cuerpo mismo del trabajo (...) una especie de instancia de libertad, incluso normativamente fundada, que puede traducirse en comportamientos quizás diversos, pero casi todos orientados hacia el desencanto» ${ }^{11}$.

De lo expuesto hasta ahora se desprenden ya algunas conclusiones a retener, como la inexistencia de un grupo juvenil ideológicamente diferenciado respecto al adulto y el delinearse de una suave evolución — sin rupturas- en la que el trabajo como valor va cediendo terreno al trabajo como instrumento, como medio para conseguir otras cosas. Desde luego, en esta evolución queda muy poco espacio para las actitudes de claro rechazo del trabajo. La relativamente extensa literatura especializada no deja lugar a dudas sobre esta cuestión. Así, sólo por poner algunos ejemplos, ya en 1980, Accornero señalaba que cada vez es más cierta la afirmación de que «entre los jóvenes no existe un rechazo del trabajo, ni como prejuicio ni como comportamiento» ${ }^{12}$. El famoso informe Schwartz redundaba en la misma dirección: los jóvenes no rechazan el trabajo, lo desmitifican. En la sociedad que conciben, los valores ya no están ligados principalmente a la producción. El trabajo ya no lleva consigo los signos del reconocimiento social debido a que está descualificado; queda excluido de los valores clave de su sistema de valores. «Sin embargo, es falso decir que los jóvenes rechazan el trabajo. Todos los estudios realizados sobre este punto coinciden en denunciar este tópico. Pero es verdad que cuestionan los modelos jerárquicos tradicionales y la parcelación de las tareas, que reivindican más autonomía en la organización del trabajo y que aspiran a reconocer en el trabajo un sentido y una utilidad. ¿Pero no son éstas las aspiraciones del conjunto de los trabajadores?» ${ }^{13}$. Entre nosotros, Moncada, aun reconociendo que los jóvenes describen el trabajo que desean con rasgos idealistas, no duda al apuntar que «de hecho se conformarían con lo que saliera, con tal de encontrar algo. Es un error muy común el afirmar que la juventud no desea trabajar, sino divertirse» ${ }^{14}$. En general, puede afirmarse que ninguna investigación empírica española ha detectado la existencia de actitudes u opiniones de rechazo cuantitativamente relevantes ${ }^{15}$. Finalmente, éstos son también los aires que se respiran en medios de la OCDE: si bien es cierto que sobre el trabajo existen opiniones muy variadas, "los temores de que "la ética del trabajo" sea rechazada por los jóvenes no parecen fundados» ${ }^{16}$. A pesar de que los empleadores

${ }^{11}$ Ibid., pp. $55-56$.

12 A. AcCornero, «I giovani e il lavoro», en Politica ed Economia, núm. 4, 1980, p. 57.

13 B. SCHWARTZ, «La inserción profesional y social de los jóvenes», en Revista de Trabajo, núm. 66,1982 , p. 367.

${ }_{14}$ A. MonCada, La adolescencia forzosa, Dopesa, Barcelona, 1977, p. 112.

is Cfr., al respecto, VV. AA., La juventud ante el trabajo. Nuevas actitudes en los 80 , E. Popular, Madrid, 1987

${ }_{16}$ OCDE-CERI, Les études et le travail vus par les jeunes, París, 1982, documento roneografiado CERI/CD (82) 12, p. 29. 
la invocan constantemente para justificar su rechazo a contratar jóvenes, la idea de que éstos tienen exigencias utópicas es errónea. «Del conjunto de datos recogidos se deduce que los jóvenes tienen una actitud crítica frente al trabajo, pero que, sin embargo, están preparados para adaptarse al mismo. Sus exigencias (...) no parecen muy diferentes a las de los adultos. (...) En la realidad, los jóvenes, como sus mayores, se acomodan al empleo que tienen» ${ }^{17}$. Pero con la crisis no se han hecho más críticos, sino más bien todo lo contrario. «Es evidente que la idea, tan extendida entre 1960 y 1980 y todavía ampliamente admitida en nuestros días, según la cual la juventud sería cada vez más refractaria al trabajo, no encaja con las conclusiones que pueden obtenerse de las encuestas realizadas entre los mismos jóvenes» ${ }^{18}$.

No se pretende con todo esto negar la posibilidad potencial de que existan tendencias hacia el rechazo o, en cualquier caso, hacia la desafección (al menos en relación con el trabajo asalariado, ya que no con el trabajo tout court). Aquí cabría preguntarse qué habría pasado con lo que podríamos llamar la "subcultura sesentayochesca», que posteriormente se ha manifestado como muchísimo menos hegemónica entre los jóvenes de lo que se temía, de no haber sido por la irrupción de la crisis económica y del empleo. Porque, desde luego, existe más de una explicación razonable que permite creer en la posibilidad de que acabe apareciendo el rechazo como fenómeno relevante. Estas explicaciones tienen como telón de fondo las transformaciones que experimenta el tejido social cuando alcanza y consolida el estadio industrial: en el sistema de valores culturales, en la esfera del consumo, en el nivel de excedente económico, en el sistema educativo, en el mercado de trabajo.

\section{Las bases objetivas del rechazo}

Durante siglos, la humanidad se ha incorporado a la actividad productiva no sólo a edad muy temprana, sino marcada por guerras, hambrunas, analfabetismo, migraciones; en una palabra, por las necesidades primarias más acuciantes. Haciendo de éstas virtud, la austeridad, el ahorro, el espíritu de sacrificio y también, a veces, el amor al trabajo, han sido otros tantos nortes de la vida social. Estos nortes han guiado incluso la primera gran larga etapa del capitalismo, la que va desde su nacimiento hasta la aparición de la producción en masa. A partir de aquí, para que la máquina pueda seguir funcionando, será necesario producir también consumidores en masa, y esto sólo es posible abriendo el sistema cultural a nuevos valores aun al precio de que entren en contradicción con los hasta entonces hegemónicos. Contradicción no fácilmente

17 Ibid., p. 34.

18 Ibid., p. 36. 
resoluble, como ha señalado Bell, ya que el sistema necesita individuos a la vez puritanos, austeros y sacrificados a la hora de producir, $\mathrm{y}$ hedonistas $\mathrm{y}$ disipados a la hora de consumir ${ }^{19}$. Según Bell, en Estados Unidos, antes incluso de la última gran guerra, los valores tradicionales, y la ética protestante entre ellos, ya habían sido arrasados por el hedonismo materialista provocado o al menos hecho posible por la llegada de la era del consumo de masas. Ahora bien, los jóvenes de las ultimísimas generaciones de las sociedades industriales avanzadas - criados en un ambiente de paz, escuela y bienestar - son, de hecho, socializados antes que todo en estos nuevos valores, pues mientras por una parte acceden al consumo cada vez más pronto - se ha consolidado ya un mercado de consumo infantil y juvenil-, por otra, su incorporación a la vida activa se hace cada vez más tarde. Dado que los códigos que rigen las conductas en ambas esferas son tan dispares, no cabe sino esperar problemas de ajuste ${ }^{20}$.

El sistema educativo, reino de la simulación de la vida, más que contribuir a la imbricación de esos códigos, complica el problema aportando el suyo propio. Desde que dejó de ser sólo una institución privilegiada, reservada a quienes ya desde la cuna habían sido redimidos de la maldición del trabajo y del sudor, para pasar a convertirse en gran parte en una obligación generalizada, dejó también de poder asegurar a todos sus usuarios una ocupación noble o al menos «a cubierto». Sin embargo, a falta de mejores argumentos con que contener en las aulas a la inquieta muchachada, esto es lo que siguen prometiendo explícitamente los pedagogos de batalla y los ilusionados padres, y lo que constituye el mensaje último de la rutinizada escolar carrera de obstáculos: que si son buenos estudiantes al final no tendrán que doblar el espinazo. Moncada, en franca contradicción consigo mismo, lo ha explicado así: «El repudio del trabajo manual, rutinario y fatigoso es una característica general de las nuevas generaciones a medida que éstas sufren más largas escolaridades, son excluidas por más tiempo del trabajo y están expuestas a los diferentes canales de información y excitación del medio industrial urbano. (...) La relación causal entre mayor educación y menor motivación para trabajos no ilustrados es clara» ${ }^{21}$. En cualquier caso, es muy posible que el sistema educativo, por su propia lógica de funcionamiento e incluso contra la voluntad de sus funcionarios, esté generando expectativas irreales, o al menos desproporcionadas, respecto a la prosaica realidad del mercado de trabajo. $\mathrm{Y}$ al resultar defraudadas estas expectativas (nuevas para los jóvenes originarios de ciertas clases sociales) aparecerían también nuevas formas de desilusión que se mani-

19 D. Bell, Las contradicciones culturales del capitalismo, Alianza, Madrid, 1977.

${ }^{20}$ En relación con los efectos de la aparición del consumo de masas sobre la sociedad española puede verse J. Castillo, «Los hijos de la sociedad de consumo española», en Revista Española de Investigaciones Sociológicas, núm. 17, 1982.

21 A. MonCADA, op. cit., pp. 209 y 210. 
festarían incluso antes de abandonar la escuela. Porque los medios de información al alcance de estos jóvenes son poderosos, y muchos de ellos saben que continúa siendo cierto que su posición al principio de la vida activa va a estar más determinada por su medio social que por su rendimiento académico, y si esto no está dando lugar en términos generales a situaciones de franca revuelta, sí puede producir estados anómicos desde los últimos años de escuela hasta los primeros de trabajo, hasta que la fragua de la vida ajuste los sueños a la realidad. Más que para alimentar el distorsionador discurso sobre la proporción que debe guardar el número de soldados con el de oficiales, esta realidad debería servir de punto de partida para una reflexión sobre las contradicciones o disfuncionalidades que introduce un sistema educativo en vías de democratización en un tejido social apoyado en un sistema productivo siempre clasista.

Pero, entonces, lo que suele valorarse como un fenómeno generacional quizás no sería más que la simple consecuencia de las transformaciones en las condiciones de escolarización y en el funcionamiento del mercado de trabajo. $\mathrm{Y}$ ese fenómeno tiene más que ver con la multiplicación del excedente económico que todo salto a la sociedad industrial entraña que con la disipación de las «buenas costumbres de antaño». El hecho de que la sociedad en su conjunto disponga de un mayor volumen de excedente económico quiere decir que una parte cada vez más importante de la colectividad, gracias a las transferencias familiares o a los mecanismos del welfare state, no depende de su propio trabajo como única fórmula de subsistencia, y, por lo tanto, que los jóvenes tienen objetivamente la posibilidad de decir que no a determinados empleos, permaneciendo a la espera de encontrar algo mejor. En opinión de Grootings, algo así está pasando ya en algunos países socialistas, donde resulta difícil encontrar jóvenes para hacer los trabajos (manuales y pesados) considerados poco atractivos: «Este fenómeno se puede relacionar en parte con el grado de industrialización: en los países socialistas, el paso de una economía agrícola a otra industrial implicó la participación masiva de los antiguos trabajadores agrícolas. En la mayoría de los países actualmente esta reserva se encuentra agotada, lo cual comporta el paso de formas de producción extensivas a formas intensivas. En aquellos países donde la transición se ha realizado recientemente (como es el caso de Bulgaria), el trabajo industrial, considerado (también por los jóvenes) como relativamente mejor que el agrícola, y su fascinación todavía no han experimentado problemas graves. En otros países (como la RDA y la URSS) parece que el problema de encontrar jóvenes que hagan faenas poco atractivas es más grave» ${ }^{22}$.

${ }^{22}$ P. Grootings, «Recerca comparativa internacional sobre el jovent $i$ el treball a Europa», en Papers, núm. 25, 1985, p. 55. 
En Emilia Romagna, una de las regiones más ricas de Europa, Capecchi ${ }^{23}$ ha detectado la existencia de ciertos grupos de jóvenes - los cuales no se sienten identificados con los postulados sindicales, aunque tampoco con los patronales- que discuten la lógica de la «estabilidad» del trabajo y su regulación, que aspiran, por ejemplo, a gestionar de otra manera el tiempo de trabajo, que pueden preferir una estrategia individualista con vistas a organizar de manera diferente la propia vida. Capecchi señala también la relación entre trabajo crecientemente degradado, población con titulaciones académicas cada vez más elevadas y mayores niveles de rotación laboral, apatía, absentismo, etc. ${ }^{24}$. Como contrapunto, en una región de pequeña empresa y espíritu empresarial generalizado como ésta, se vislumbran tendencias hacia el trabajo autónomo que tienen su punto de partida en el rechazo del trabajo degradado y, más en general, del trabajo dependiente. Así, trabajadores cualificados entre los 30 y los 40 años de edad se establecen por su cuenta, entre otras cosas, para tener más autonomía. En el caso de los jóvenes estas tendencias asumen a veces la forma del recurso a la economía sumergida (en este caso rica, como la región) en busca de trabajos más profesionalizados o que permitan mayor autonomía al menos en uno de sus aspectos: la gestión de su duración. Sin embargo, concluye Capecchi, el elevado porcentaje de jóvenes que de una u otra manera combinan el estudio con el trabajo, así como la tendencia (más pronunciada entre los estudiantes de institutos técnicos industriales) a abandonar los estudios cuando se presentan oportunidades de empleo, pone en aprietos la tesis del rechazo del trabajo.

Aunque puede afirmarse que la institución del aprendizaje sigue existiendo, ahora en forma de ocupaciones precarias en el mercado secundario, en régimen de economía sumergida o no, sin embargo, también es cierto que las modalidades de incorporación al mundo del trabajo se han modificado profundamente, sobre todo, pero no sólo, en relación con los jóvenes originarios de clase obrera. Una idea de lo que significan estos cambios respecto a la situación anterior, en cualquier caso relativamente reciente, puede deducirse del siguiente texto citado por Merle: «Todavía hacia 1950, la mayoría de los jóvenes entraba en la vida activa a los 14 años. Muchos no tenían ni siquiera la posibilidad de elegir el lugar de entrada: hijos de agricultores permaneciendo en la granja hasta el momento del servicio militar o de la mayoría de edad,

${ }^{23}$ V. CAPECCHI, «Lavoro e condizione giovanile», en Problemi della Transizione, núm. 4, 1980; particularmente, pp. 35, 49, 58 y 59.

${ }^{24}$ Una situación similar vuelve a encontrar este mismo autor en una investigación más reciente sobre los jóvenes de un barrio periférico de Bolonia, realizada junto con A. Pesce; es decir, la existencia de una relación muy estrecha entre trabajos precarios realizados por los jóvenes, mayores niveles de escolaridad y proyectos de establecerse por su cuenta, proyectos que, aun cuando después en muchos casos no podrán ser realizados, traslucen el deseo de no caer en el rrabajo dependiente. Vivere lo spazio e il tempo, Comune di Bologna, 1986. 
hijos de artesanos y de comerciantes aprendiendo el oficio con los padres. Otros conocían el aprendizaje o el trabajo asalariado precoz. La adolescencia era vivida en el trabajo; el largo período de maduración, de descubrimiento de uno mismo y de los otros, la expresión de los deseos y de los rechazos estaban así ligados a la actividad en unas empresas que, contrariamente a cuanto ocurre en la actualidad, sin duda, hacían sitio al joven como tal» ${ }^{25}$. Durante siglos, la gran mayoría de los jóvenes han tenido que seguir el oficio del padre e incluso del abuelo; se han visto permeados, por tanto, de la cultura del trabajo ya en la propia familia. Este mecanismo de transmisión de conocimientos y habilidades se traba con la profundización de la industrialización, la aceleración del cambio tecnológico, la degradación del trabajo y la generalización del paro. El padre ya no quiere que el hijo aprenda su oficio, sino todo lo contrario, que vaya a la escuela para que pueda ganarse la vida menos duramente que él; pero es que, aun cuando lo quisiera, las posibilidades de que esto sea así se hacen cada vez más escasas: en los sectores con paro estructural no tiene sentido la transmisión, muchos oficios no duran ya lo que la vida activa de un hombre, y otros tantos no son sino una burda caricatura de lo que eran. Difícilmente puede alguien sentirse interesado por la ejecución de una tarea vaciada de contenido. $Y$ cuando al abandonar la escuela el futuro obrero se enfrenta ante ella, es muy fácil que llegue a pensar que para ese viaje no hacían falta esas alforjas. Porque, a pesar de las apreciaciones de Cottereau, gusto por el trabajo e interés por la técnica también debe haberlos habido, tanto entre los veteranos como entre los jóvenes obreros. No debe haber sido demasiado excepcional la situación que hace ya tiempo describía Mallet: «Hace un año la dirección (...) ponía a punto el tambor magnético. Un equipo de jóvenes vino a trabajar el sábado por la mañana y por la tarde sin que nadie se lo hubiese pedido, sino sencillamente porque tenían prisa en ver cómo funcionaba el maravilloso aparato. Volvieron a la fábrica la noche del sábado al domingo, continuando toda la jornada del domingo y la noche, ¡dándose cuenta de que no habían comido a mediodía! Un joven, a quien el delegado sindical pedía detalles sobre el salario, se encogió de hombros y respondió: "Me importan poco estas historias de salarios; lo que me interesa aquí es la técnica" ${ }^{26}$.

En el nivel material de la sociedad, con la desaparición del oficio y la degradación de las tareas, se ponen las bases objetivas para la aparición de actitudes refractarias ante el trabajo; en el nivel cultural, con la llegada de la sociedad de consumo y la cultura de masas, desaparece la cultura obrera, y,

${ }^{25}$ V. Merle, «Desenchantement plus qu'allergie», en Autrement, núm. 21, 1979, p. 19. Este número de Autrement, íntegramente dedicado a jóvenes y trabajo y basado en unas sesenta historias de vida, ofrece numerosos ejemplos de negativas a aceptar determinados trabajos degradados.

${ }^{26}$ S. Mallet, La nueva condición obrera, Tecnos, Madrid, 1969, p. 86. 
por tanto, la inculcación en el seno de la familia obrera de determinadas formas de entender la vida y el trabajo, con lo que se refuerza la posibilidad de que germinen aquellas mismas actitudes. Porque hasta hace poco la condición obrera expresaba tanto una relación de producción, una situación dentro de la fábrica, como un modo de vida social, una manera de vestir, de consumir, de ocupar el tiempo libre, unos determinados barrios y viviendas... Dicho brevemente: una cultura obrera. Todo este mundo, bien captado en una película ya añeja de Pietro Germi - Il ferroviario-, está en trance de desaparición; la vida cotidiana de la clase obrera ha dejado de ser un comportamiento sociológico especial. En la sociedad postindustrial, sobre todo en las aglomeraciones urbanas, tiende a establecerse un continuum, si no entre las formas de trabajo, entre las formas de consumo de la clase obrera y las clases medias; la vinculación entre la forma en que se ejerce el trabajo y la cultura y formas de vida a él asociadas tiende a relajarse. Las viejas formas de socialización obrera se baten en retirada ${ }^{27}$. Podría adelantarse la hipótesis, por tanto, de que, en las condiciones actuales de trabajo y de vida, la interposición entre la familia y la unidad productiva de instituciones como la escuela prolongada o el mercado de consumo juvenil amplía objetivamente la posibilidad de que se manifiesten actitudes refractarias. Lo que no sería sino la otra cara de la consolidación del estado adolescente como fenómeno sociológico relevante. Como ha señalado Accornero, las sociedades capitalistas avanzadas no han sido capaces de ofrecer a las nuevas generaciones un trabajo conforme a las expectativas y a la titulación académica, y a veces ni siquiera un trabajo cualquiera; han separado progresivamente el sistema educativo del productivo, enfrentándolos en vez de sintonizarlos, y han desplazado el baricentro social desde la producción al consumo, cuestionando la centralidad y la credibilidad del valor trabajo ${ }^{28}$. En estas circunstancias, el rechazo del trabajo podría ser interpretado también como un mecanismo de defensa psicológica ante la escasez cuantitativa y cualitativa de empleos; y, además, como resultado, por una parte, de la opinión de que para el trabajo degradado ya están las máquinas y, por otra, del descrédito del concepto de jerarquía-autoridad sobre el que se basa la organización del trabajo.

\section{El rechazo es minoritario}

Sin embargo, la evidencia empírica muestra no sólo que estas actitudes no son mayoritarias, aunque a veces atañan a minorías dignas de considera-

${ }^{27}$ Un análisis más detenido de esta cuestión puede encontrarse en Y. CLot, «Jeunesse, travail, société. Voies et enjeux d'une mutation», en La Pensée, núm. 225, 1982.

${ }_{28}$ A. Accornero, Il lavoro come ideologia, Il Mulino, Bolonia, 1980, pp. 11-14. 
ción, sino que el trabajo continúa siendo un elemento muy importante en la vida de los jóvenes. (Que esto sea así por motivos meramente instrumentales u otros más nobles es otra cuestión.) Dan buena prueba de ello, por ejemplo, diversas investigaciones sobre colectivos de jóvenes en paro realizadas utilizando la técnica de la encuesta o la de las historias de vida, donde puede apreciarse la forma, a veces dramática, como muchos de ellos viven esta situación.

A partir de doscientas entrevistas en profundidad realizadas en 1979 a jóvenes parados franceses - no licenciados superiores y menores de 25 años-. Louis y Galland ${ }^{29}$ los han clasificado en los tres tipos ideales siguientes. En primer lugar están los parados vergonzosos, hijos de obreros cualificados cuya cultura se encuentra muy marcada por el trabajo y el reconocimiento de las capacidades profesionales. Para ellos el paro significa ruptura, negación individual y negación de una comunidad constituida por y para el trabajo. Rechazan el paro y están incapacitados para asumir positivamente tal situación. El tiempo de paro no puede ser vivido como tiempo libre y sólo puede ser ocupado por la búsqueda de empleo o la reclusión voluntaria. Buscan un empleo en concordancia con su cualificación, pero aceptan lo que sea con tal de trabajar, aunque sin perder de vista su objetivo. Por este motivo es posible que cambien de trabajo con frecuencia, actitud que puede interpretarse en términos de «movilidad de resistencia». El segundo grupo es el más inclinado a adoptar actitudes de rechazo: hijos de clases medias con titulaciones secundarias desvalorizadas en el mercado de trabajo y bloqueados en sus posibilidades de realización profesional y ascenso social, y también hijos de los estratos decadentes de la clase obrera (ocupados en oficios y/o industrias en regresión, etc.). En este grupo se produce una distancia irreductible entre los proyectos personales y las posibilidades concretas de ponerlos en práctica a través de un empleo. Aceptan trabajos sin ningún interés por motivos puramente instrumentales, como viajar o comprarse la moto; y cuando no existe un objetivo concreto de este tipo les resulta muy difícil soportar el trabajo, aunque en realidad no rechazan tanto éste como la disciplina y orden jerárquico que entraña. Aquí el despido o el paro ya no son vividos como una situación de fracaso, sino con indiferencia e incluso con alivio. El paro ya no es la versión catastrófica del trabajo, ni siquiera se define en relación con el trabajo; tiene un valor en sí mismo: «es la inversión de la relación tradicional vida cotidiana/trabajo, en la cual la primera no es más que el residuo de un tiempo dominado" ${ }^{30}$. Por último, en tercer lugar se encontrarían aquellos jóvenes de extracción obrera situados en la periferia no sólo del mercado de trabajo, sino

${ }^{2} \mathrm{M}$. V. Lours, «Actitudes de los jóvenes parados respecto al trabajo», en De Juventud, núm. 15, 1984. Basado también en historias de vida de jóvenes parados franceses, puede consultarse, igualmente, G. Balazs, «Les facteurs et les formes de l'expérience du chômage», en Actes de la Recberche en Sciences Sociales, núm. 50, 1983.

${ }^{30}$ Ibid., p. 65. 
del sistema social (mujeres, inmigrantes, minusválidos), para quienes el trabajo es el medio a través del cual pueden integrarse en la sociedad. Estos no rechazan nada, están dispuestos a trabajar en lo que sea.

Se desprende de todo esto algo muchas veces subrayado por la literatura francesa: fenómenos como el absentismo, la elevada rotación o la desafección asumen significados muy diversos en función de la clase social de origen. En todos los casos - siempre en relación con la minoría de desafectos, que podría estimarse en un 30 por 100 si por tales entendemos los partidarios de trabajar a tiempo parcial porque les ofrece más libertad y les permite escoger su propia manera de vivir ${ }^{31}$ - nos encontramos ante una estrategia de prolongación de la adolescencia, porque lo que se rechaza no es tanto la ideología del trabajo como la forma tradicional de entender la vida adulta. Pero esta estrategia de diferir la incorporación al trabajo, para los jóvenes de clases sociales más elevadas, puede interpretarse como un intento de escapar al descląsamiento buscando el instalarse en nuevas profesiones todavía socialmente poco definidas, pero con una imagen equivalente a la de las profesiones tradicionales. Por el contrario, para los jọvenes de clases menos elevadas, se trata sobre todo de huir de la explotación, adoptando modos de vida marginales hasta que la paulatina asunción de las responsabilidades adultas ya no lo permita.

Una investigación muy interesante de Blanch, realizada en 1985 entre jóvenes (16-25 años) del área metropolitana de Barcelona, nos conduce justo a las antípodas del discurso sobre la alergia al trabajo ${ }^{32}$. Apoyándose en resultados de investigaciones realizadas en distintos países, Blanch comienza afirmando que en las sociedades occidentales el trabajo sigue percibiéndose como un valor de primer orden, la seña de identidad más característica, el eje vertebrador de creencias, actitudes y opciones fundamentales. Esta situación se aprecia también entre los jóvenes estudiados, quienes conceden al trabajo un alto valor y para los cuales el empleo tiene un gran atractivo. Aunque la investigación no permite saber con certeza si el valor atribuido al trabajo es instrumental o final, sin embargo, hay indicios de que el empleo sigue representando algo más que una simple fuente de financiación de la existencia, de que sigue siendo un valor más que instrumental ${ }^{33}$. Coherentemente con todo esto, «el joven desempleado barcelonés tiende a experimentar la vida de modo más depresivo que el empleado». Esa experiencia deprimente se apoya, por una parte, en el problema económico subyacente y, por otra, en el problema moral: el paro afecta a la autoimagen, a la autoestima y al sentido de la propia identidad personal y social. Precisamente en relación con el problema moral «se puede

31 Cfr. O. Galland, Les jeunes, La Decouverte, París, 1984, p. 99.

${ }^{32}$ J. M. Blanch, Desempleo juvenil y salud psicosocial, Documentos de Psicología Social, Universidad Autónoma de Barcelona, 1986.

${ }^{33}$ Ibid., pp. 50 y ss. 
hablar con propiedad de un efecto sobredepresivo asociado a la experiencia del desempleo» ${ }^{34}$. Tan es así, y esto asombrará a muchos, que Blanch acaba recomendando la lucha contra ese lugar preponderante que ocupa el trabajo en la escala de valores de los jóvenes como terapia preventiva de salud mental.

Los datos sobre niveles de satisfacción laboral entre los jóvenes ocupados ponen también en aprietos la tesis del rechazo. Los que maneja Galland, relativos a Francia, le permiten afirmar que entre dos terceras y tres cuartas partes parecen siempre satisfechas de su trabajo, situación que ha evolucionado poco entre 1960 y $1975^{35}$; siendo, además, siempre superior al 50 por 100 el número de jóvenes que entre 1966 y 1978 están dispuestos a hacer sacrificios para comenzar bien su vida profesional. Por lo que se refiere a Italia, Romagnoli subraya cómo, a pesar de las duras y malas condiciones de trabajo, sóló poco más de la cuarta parte de los jóvenes ocupados se manifiesta insatisfecha con su trabajo, mientras que un porcentaje algo superior se declara muy satisfecho y el 45 por 100 bastante satisfecho. Y esto a pesar también de que el 47 por 100 estima que desarrolla una actividad muy por debajo de su preparación. De donde deduce Romagnoli que lo que parece predominar es una actitud de gran cautela y prudencia en la valoración de la propia condición: cada cual busca en el trabajo sólo aquello que éste puede dar, es decir, no la autorrealización -altamente improbable en trabajos prevalecientemente manuales y escasamente cualificados-, sí mayores ingresos $\mathrm{y}$, sobre todo, condiciones más aceptables ${ }^{36}$.

La situación en España es similar: dos de cada tres jóvenes se encuentran muy o bastante satisfechos con su trabajo ${ }^{37}$; además, en 1982 , el 59 por 100 de ellos prefería un buen empleo a seguir estudiando, frente al 38 por 100 que se manifestaba en sentido contrario. Esta escala de preferencias resulta prácticamente la inversa de la correspondiente a 1977, por lo que puede presumirse que la escalada del paro ha debido desempeñar su papel. De hecho, García Ferrando señala que «prácticamente tres de cada cuatro jóvenes españoles que buscan trabajo desearían que éste fuera estable y fijo, lo que parece contradecir, para el caso español, la explicación culturalista del desempleo juvenil que se ha dado para otras sociedades» ${ }^{38}$. Y Montoro, en las escasas páginas que dedica al tema de la satisfacción en el trabajo, abunda en la misma dirección y llega incluso más allá subrayando el elevado grado de sociabilidad

${ }^{34}$ Ibid., pp. 69-70.

${ }^{35}$ O. Galland, Les jeunes, cit., p. 97.

${ }^{36} \mathrm{G}$. Romagnoli, op. cit., p. 75.

${ }^{37}$ M. García Ferrando, «Situación laboral de la juventud española», en VV. AA., Informe sociológico sobre la juventud española, cit., p. 43.

38 Ibid., p. 50.

${ }^{39}$ R. MONTORo, La inserción en la actividad económica: empleo y paro juvenil, Ministerio de Cultura, 1985, p. 168. 
laboral de los jóvenes respecto a sus jefes y compañeros: «los jóvenes que tienen la oportunidad de trabajar componen un colectivo bastante disciplinado laboralmente hablando, con altos índices de sociabilidad laboral, con deseos de trabajar y con un grado de satisfacción en el trabajo muy elevado" ${ }^{39}$ Ahora bien, si convenimos que los jóvenes no son masoquistas podremos aceptar que más que satisfacción en el trabajo lo que hay es satisfacción por haber tenido la suerte de encontrar un hueco en el mercado de trabajo. En este sentido, la conclusión de un estudio de López Jiménez sobre los jóvenes trabajadores del casco antiguo de Zaragoza parece absolutamente plausible: por necesidad aceptan unas condiciones de trabajo insatisfactorias, pero manifiestan una rebeldia latente ante unas tareas que sólo contribuyen a garantizar la subsistencia. No es el hecho de trabajar lo que se cuestiona, sino qué trabajo y cómo se hace ${ }^{40}$. Posiblemente, al menos mientras dure la crisis del empleo, la rebeldía latente no pasará de eso.

\section{Trabajo valor y trabajo instrumento}

Visto que los jóvenes no sólo no rechazan el trabajo, sino qie incluso le conceden un alto valor, queda una última cuestión por abordar, ya varias veces sugerida en estas páginas: si esto es así por motivos sólo instrumentales o por algo más. La literatura al respecto suele ser, si no confusa, al menos no convergente. El mismo pasaje del informe Schwartz antes citado, mientras, por una parte, señala que los jóvenes desmitifican el trabajo, por otra, afirma que también aspiran a encontrar en él un sentido y una utilidad. Dicho en otras palabras, y creo que sin forzar los términos, por una parte, ya no quieren hacer del trabajo el centro de sus vidas (actitud instrumental) y, por otra, aspiran a trabajar por algo más que sólo dinero (actitud finalista). Y, de hecho, una parte significativa de las investigaciones en la materia, tras descartar la hipótesis del rechazo, acaban poniendo el acento, bien sobre las inclinaciones crecientemente instrumentalistas de los jóvenes, bien sobre la mayor importancia que conceden al contenido del trabajo (que sea interesante, que merezca la pena, etc.). Probablemente, esta paradoja se engendra ya en la misma metodología y técnicas de investigación. De un mismo sistema de valores pueden derivar constelaciones de opiniones no necesariamente sólidas - como bien sabe la sociología electoral-, ni tampoco necesariamente coherentes entre sí ni respecto a aquél. Por añadidura, una opinión no tiene por qué traducirse obligatoriamente en una actitud, y las mismas opiniones pueden, incluso, ir combinadas con actitudes diametralmente opuestas, y viceversa.

${ }^{40}$ M. A. López JimÉnEZ, «Consideraciones sobre la actitud de los jóvenes ante el trabajo», en De Juventud, núm. 9, 1983, y, más recientemente, Los bienatados. Jóvenes en el casco viejo de Zaragoza, vol. II, Institución Fernando el Católico, Zaragoza, 1987. 
La constatación de un mismo hecho objetivo - la tacañería y mezquindad del mercado de trabajo- puede dar lugar a toda suerte de interpretaciones, siempre legítimas, sobre el tema que nos ocupa: que el trabajo, como cualquier bien, se revaloriza cuando escasea; que esta revalorización sólo puede entenderse en términos instrumentales porque la calidad de lo que ofrece el mercado no merece otra cosa; que ello no tiene demasiado sentido en una sociedad de relativo bienestar donde ya no es necesario trabajar para vivir; que los graves problemas psicológicos que el paro entraña muchas veces sólo se explican en relación con una concepción más que instrumental del trabajo; que es a la crisis y no a la sociedad de consumo a la que hay que atribuir unos supuestos cambios generalizados (no sólo entre los jóvenes) en la cultura del trabajo, que está cediendo el paso a la cultura del puesto y del subsidio: lo importante no es en qué se puede trabajar, sino meter la cabeza en algún sitio, y al final ya no sabe si lo que se reclama es un trabajo, un puesto o una renta... Son interpretaciones legítimas todas ellas probablemente porque las técnicas al alcance del investigador social sólo permiten todavía aproximarse con dificultad a esta tierra de nadie donde hechos sociales y comportamientos individuales escapan a la hermenéutica sociológica y psicológica.

La paradoja se hace patente al contrastar varias investigaciones italianas recientes. De una encuesta administrada a una muestra de 1.200 jóvenes napolitanos, D'Alessandro ${ }^{41}$ extrae las siguientes conclusiones. $\mathrm{Ha}$ aparecido un auténtico nuevo ethos juvenil en relación con el trabajo, en el contexto del cual éste sigue estando presente, pero indiscutiblemente relativizado: es algo importante, pero no la vía principal de realización; los jóvenes no rechazan el trabajo, pero se muestran mucho más realistas ante el mismo de lo que lo fueron las generaciones inmediatamente anteriores cuando tenían su edad; en consecuencia, se adaptan casi espontáneamente a un sistema social en el que el trabajo es cada vez menos importante. En definitiva, para D'Alessandro, estamos asistiendo a la transición desde una cultura "mediterránea» hacia una actitud «postindustrial» en relación con el trabajo. Sólo algo más del 20 por 100 de los 500 individuos — no jóvenes y ocupados en distintas fábricas del Norte y Sur, con cualificaciones medio-bajas- que constituyen la muestra de otra investigación habla todavía del valor moral del trabajo, mientras que todos los demás resaltan su carácter instrumental; y sólo menos del 5 por 100 considera que el trabajo representa el valor principal de la vida ${ }^{42}$. Los italianos con más de una ocupación — colectivo que se cuenta por millones- parecen ser quienes albergan en mayor medida las concepciones instrumentales ${ }^{43}$. Romagnoli, que encuentra un 68 por 100 de jóvenes que consideran el trabajo

${ }^{41}$ V. D'Alessandro, Etbos giovanile e lavoro, Franco Angeli, Milán, 1985.

${ }^{42}$ M. La Rosa (ed.), Il lavoro che cambia, Ftanco Angeli, Milán, 1985.

${ }^{43}$ L. Gallino (ed.), Il lavoro e il suo doppio, Il Mulino, Bolonia, 1985. 
como un elemento muy importante en la propia existencia (inmediatamente después de la familia y por delante de las relaciones afectivas), interpreta, no obstante, que si el trabajo cuenta mucho lo es, sobre todo, como realidad concreta y como instrumento: en la imagen del trabajo prevalecen los elementos vinculados a la prestación sobre los vinculados al valor. Hay, por tanto, un realismo de fondo frente al trabajo, y se va configurando una imagen del mismo basada casi exclusivamente en elementos de tipo laico-racional. No hay una particular adhesión al trabajo como valor, «quizás sólo es una "parte de la vida", no el objeto principal a través del cual pasa la catarsis revolucionaria, ni tampoco la vía maestra de la autorrealización»" ${ }^{44}$. Sin embargo, Garelli, que a pesar de trabajar con datos de otra encuesta obtiene resultados similares a los de Romagnoli, llega a conclusiones diversas.

Para Garelli, hay básicamente dos interpretaciones de la actitud de los jóvenes frente al trabajo. Según la primera, más tradicional, el trabajo ya no es portador de aquellos valores tradicionales - -éxito, carrera, ingresos - por los que era considerado como vehículo de status social, como canal de mejora de las condiciones sociales del individuo y de la familia de origen. Ante la imposibilidad de experimentar tales metas en el trabajo, los jóvenes reaccionan con una actitud de desafección que conduciría o al rechazo o a una aceptación puramente instrumental, no buscando en el trabajo más que un medio de vida. Este empobrecimiento de la esfera laboral se compensaría por la búsqueda en la esfera privada de aquello que no se encuentra en el trabajo. Esta sería la interpretación convencional de las actitudes de los jóvenes contestatarios de los años sesenta y setenta. Para la segunda interpretación, más reciente, el punto de partida nó es la imposibilidad por parte de los jóvenes de hacer «carrera social» en el trabajo, sino un cambio en la sensibilidad de los jóvenes que les llevaría a preocuparse más por el contenido concreto del trabajo que por los aspectos instrumentales, buscando una calidad de vida construida también a partir de la experiencia laboral. Más que huida del trabajo hacia el área del consumo y del tiempo libre para buscar allí el sentido de la vida, lo que habría sería una continuidad de esa búsqueda de sentido entre uno y otros ámbitos. Garelli lee sus resultados en línea con la segunda interpretación. Son los siguientes: el 2,6 por 100 manifiesta actitudes de rechazo; el 17,6 por 100, instrumentales; para el 60 por 100 el trabajo es uno de los aspectos más importantes de la vida, y para casi el 20 por 100 es incluso el más importante ${ }^{45}$. De donde deduce, coincidiendo estrechamente con las conclusiones de Blanch, que existe una identificación generalizada con el trabajo ${ }^{46}$ que se man-

4 G. Romagnoli, op. cit., p. 78.

${ }^{45} \mathrm{~F}$. Garelli, La generazione della vita quotidiana, Il Mulino, Bolonia, 1984, p. 149.

${ }^{46}$ Conclusiones similares para el caso inglés pueden encontrarse en L. J. Frances, Youth in Transit. A profile of 16-25 years olds, Gower, Londres, 1982. 
tiene incluso por encima de las diferencias que pueden observarse entre los distintos tipos de jóvenes. Por ejemplo, el porcentaje de jóvenes que rechaza el trabajo oscila entre 1 y 5 , y el de quienes mantienen actitudes instrumentales entre 10 y 25 . Por tanto, la gran mayoría ni rechaza el trabajo ni lo valora únicamente en términos instrumentales; aprecia más otros factores como la existencia de un buen ambiente de trabajo y que éste sea interesante.

Es posible que esto sea así, pero, en cualquier caso, no parece tarea fácil delimitar con nitidez dónde acaban las concepciones instrumentales y dónde comienzan las que algunos autores han denominado expresivas (el trabajo como búsqueda de libertad, autonomía, creatividad, etc.), pues, probablemente, todos - jóvenes o no- nos movemos de manera ambigua entre unas y otras. Pero hay otras conclusiones de Garelli —en terreno menos resbaladizo- que me parecen significativas, y son las relacionadas con determinadas diferencias que se aprecian cuando se distingue entre unos y otros jóvenes. Por ejemplo, al pasar del estudiante al ocupado los planteamientos frente al trabajo parecen hacerse más realistas: se da menos importancia al interés, cualificación o utilidad social del trabajo, y más a cuestiones como la solidaridad con los compañeros y la seguridad ambiental; los ocupados tienen mayor tendencia a buscar un trabajo de poca responsabilidad y pocas horas. En definitiva, «la experien. cia laboral aumenta la probabilidad de madurar una desafección respecto al trabajo ${ }^{47}$, de donque, quizás, puede deducirse, razonando a la inversa, que no es a causa del rechazo por lo que los jóvenes parados no encuentran empleo. Sin embargo, los ocupados en precario tienden a manifestar más las actitudes consideradas típicamente juveniles, deseando un trabajo que les deje tiempo libre, etc. Quizás tratan de combinar la precariedad laboral con la realización personal fuera de ese ámbito, aunque -apunta Garelli- no se sabe si esta orientación es intencional o forzada, si es el resultado no querido de una situación de marginación objetiva o forma parte de una estrategia válida para los años juveniles y destinada a perder entidad con el paso del tiempo. Parece que la situación de precariedad está a medio camino entre la adaptación a condiciones objetivas y la orientación a no identificarse completamente con un modelo de realización profesional. En todo caso, estas observaciones no son de aplicación a los parados, que lo están porque no tienen otra posibilidad.

Por lo que se refiere a la situación francesa bastará con señalar que los últimos datos de que se ha podido disponer -correspondientes a un sondeo SOFRES entre jóvenes de 13 a 17 años ${ }^{48}$ - indican, por una parte, que el miedo al paro va en aumento: el 56 por 100 le teme mucho, mientras que

47 E. GARELli, op. cit., p. 155.

${ }^{48} \mathrm{Cfr}$. Le Nouvel Observateur, núm. 1116, marzo 1987. 
en 1978 esta situación afectaba al 42 por 100 ; y, por otra, que encontrar un trabajo interesante sigue siendo una preocupación mayoritaria: a la pregunta cuáles son las dos o tres cosas más importantes para ti en este momento, el 53 por 100 sitúa en primer lugar esta preocupación, por delante de cuestiones como la felicidad familiar, los viajes, la música, el dinero o la seguridad. De hecho, desde 1966 existe una recalcitrante mayoría de jóvenes franceses en cuya opinión lo más importante para ser felices es el trabajo interesante, disfrutar en la profesión, antes que en la familia o en el tiempo libre.

Finalmente, entre los estudiosos españoles, la creencia de que priman las concepciones instrumentales está tan interiorizada que muchas veces se da por descontado que esto es así y se pasa directamente a intentar explicarlo razonablemente ${ }^{49}$. La siguiente manifestación, recogida por Moncada de labios de un estudiante de ingeniería, hijo de un industrial vasco, refleja muy bien en lenguaje llano este sentir: «Cuando yo estaba en el taller, casi un verano entero, notaba que la gente mayor sentía el trabajo como suyo, mientras que los jóvenes no. Los mayores han vivido la historia de la empresa y todos han prosperado y no les importa arrimar el hombro. Los jóvenes van al tajo de otra manera» ${ }^{50}$. Sin embargo, ya hemos podido ver cómo difieren de todo esto los datos que maneja Blanch y las conclusiones que de ellos extrae. Y una encuesta piloto realizada sobre una muestra de 203 estudiantes de último curso de universidad y FP2, supongo que del área de Madrid, con vistas a una investigación más amplia, refuerza la tesis de Blanch. Se intentó medir el peso de cinco posibles orientaciones hacia el trabajo: estándar de excelencia, pasotismo, instrumental-económica, integrativo e independencia. Pues bien, los valores más altos resultaron ser independencia, estándar de excelencia e integrativo, y los más bajos instrumental y pasotismo ${ }^{51}$. En definitiva, como ha señalado Torregrosa, «no puede decirse que se haya producido una desvalorización o pérdida de importancia subjetiva del trabajo entre los jóvenes españoles. Más bien parece que, por el contrario, lo que se ha producido es una revalorización del mismo. Una revalorización instrumental en primer lugar, es cierto; pero que también aspira a ser expresiva e integrativa» ${ }^{52}$.

No se pretende con todo esto decir que el tema esté cerrado, pero sí, al menos, advertir que, desde la perspectiva que aquí hemos tomado, puede resultar tremendamente injusto $\mathrm{y}$, además, científicamente pretencioso hacer re-

4 Cfr., a este respecto, particularmente la obra colectiva citada en la nota 15.

so A. Moncada, op. cit., p. 149.

${ }^{51}$ VV. AA., Actitudes y valores de los jóvenes ante el trabajo, CIDE, Ministerio de Educación y Ciencia, 1982, mecanografiado.

$52 \mathrm{~J}$. R. Torregrosa, «Actitud de los jóvenes ante el trabajo: una interpretación desde datos de encuesta», comunicación presentada al Simposio sobre Juventud, Trabajo y Desempleo, Toledo, diciembre 1986 (en prensa). 
caer sobre los jóvenes parados la responsabilidad de encontrarse en esa situación. En las condiciones actuales de paro masivo, antes de preguntarse si las víctimas son también culpables, resulta mucho más pertinente plantearse por qué existe esa diferencia abismal entre empleos disponibles y candidatos a ocuparlos. 